\section{BRAZIULIAN JOURNAL}

OF MEDICAL AND BIOLOGICAL RESHARCH

www.bjournal.com.br
ISSN 0100-879X

Volume 43 (8) 698-811 August 2010

BIOMEDICAL SCIENCES

AND

CLINICAL INVESTIGATION

Braz J Med Biol Res, August 2010, Volume 43(8) 777-785

doi: 10.1590/S0100-879X2010007500069

Effect of auditory training on the middle latency response in children with (central) auditory processing disorder

E. Schochat, F.E. Musiek, R. Alonso and J. Ogata

The Brazilian Journal of Medical and Biological Research is partially financed by
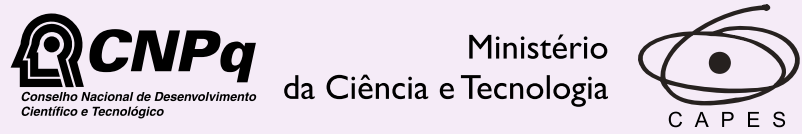

Ministério da Educação

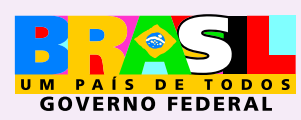

DFAPESP

Institutional Sponsors
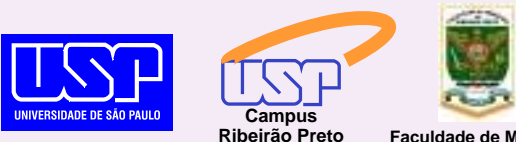

Ф SHIMADZU

GE Healthcare
Hotsite of proteomics metabolomics developped by: 


\title{
Effect of auditory training on the middle latency response in children with (central) auditory processing disorder
}

\author{
E. Schochat ${ }^{1}$, F.E. Musiek ${ }^{2}$, R. Alonso ${ }^{1}$ and J. Ogata ${ }^{1}$ \\ 1Departamento de Fisioterapia, Fonoaudiologia e Terapia Ocupacional, Faculdade de Medicina, \\ Universidade de São Paulo, São Paulo, SP, Brasil \\ 2Department of Communication Sciences, University of Connecticut, Storrs, CT, USA
}

\begin{abstract}
The purpose of this study was to determine the middle latency response (MLR) characteristics (latency and amplitude) in children with (central) auditory processing disorder [(C)APD], categorized as such by their performance on the central auditory test battery, and the effects of these characteristics after auditory training. Thirty children with (C)APD, 8 to 14 years of age, were tested using the MLR-evoked potential. This group was then enrolled in an 8-week auditory training program and then retested at the completion of the program. A control group of 22 children without (C)APD, composed of relatives and acquaintances of those involved in the research, underwent the same testing at equal time intervals, but were not enrolled in the auditory training program. Before auditory training, MLR results for the (C)APD group exhibited lower C3-A1 and C3-A2 wave amplitudes in comparison to the control group [C3-A1, $0.84 \mu \mathrm{V}$ (mean), 0.39 (SD - standard deviation) for the (C)APD group and $1.18 \mu \mathrm{V}$ (mean), 0.65 (SD) for the control group; C3-A2, $0.69 \mu \mathrm{V}$ (mean), 0.31 (SD) for the (C)APD group and $1.00 \mu \mathrm{V}$ (mean), 0.46 (SD) for the control group]. After training, the MLR C3-A1 [1.59 $\mu \mathrm{V}$ (mean), 0.82 (SD)] and C3-A2 [1.24 $\mu \mathrm{V}$ (mean), 0.73 (SD)] wave amplitudes of the (C)APD group significantly increased, so that there was no longer a significant difference in MLR amplitude between (C)APD and control groups. These findings suggest progress in the use of electrophysiological measurements for the diagnosis and treatment of (C)APD.
\end{abstract}

Key words: Auditory processing; Auditory training; Central auditory processing disorder; Evoked potentials; Middle latency response

\section{Introduction}

Many studies have used electrophysiological measurements to objectively investigate auditory processing disorders (1-3). Unlike speech and other behavioral auditory processing tests, auditory-evoked potentials can often be recorded regardless of a child's developmental age, language, motivation, or attention level. Using these potentials, auditory processing from the level of the eighth nerve to the auditory cortex has been investigated using the auditory brainstem response (ABR) and middle latency response (MLR), as well as the late cortical, P3, and mismatch negativity (MMN)-evoked responses (1-4).

The American Speech-Language-Hearing Association (ASHA) Task Force on (Central) Auditory Processing (5) has concluded that electrophysiological measures are useful for the diagnosis of (central) auditory processing disorders [(C)APDs], but has also acknowledged that further research is needed to establish the clinical utility of middle and late evoked potentials. More recently, the Bruton Conference held at the Callier Center in Dallas (6) recommended that a minimal test battery for the diagnosis of (C)APD in schoolage children should include ABR and MLR testing.

In addition to assessing the status of a child's auditory processing abilities at a single clinical visit, auditory-evoked potentials can also be used to monitor changes in central auditory function over time. There is emerging evidence that auditory-evoked potentials can be used as an objective, noninvasive tool to investigate auditory processing and plasticity of auditory function in humans (2,7-11). Kraus et al. (12) observed that listeners with normal hearing who received extensive training in a subtle speech contrast task had corresponding changes in the MMN elicited by these speech differences, as well as improvements in behavioral discrimination performance. In a subsequent study, the improvements in performance and MMN after training

Correspondence: F.E. Musiek, 850 Bolton Road, Storrs, CT 06269, USA. Fax: +1-860-486-3166. E-mail: Frank.musiek@uconn.edu

Received August 9, 2009. Accepted June 29, 2010. Available online July 23, 2010. Published August 13, 2010. 
were shown to generalize to other related stimuli (13). Expanding on previous study, Menning et al. (14) found that after auditory training in a frequency discrimination task, behavioral performance in the frequency discrimination task improved, and the amplitudes of both $\mathrm{N} 1$ and MMN increased.

Klein et al. (15) used ABR, MLR, and cortical eventrelated potentials (ERP) to analyze their subjects with verbal auditory agnosia. They used both tones and consonant-vowel stimuli presented in an oddball discrimination paradigm (for the cortical potentials). These subjects exhibited normal ABRs and MLRs and abnormalities only in the cortical ERP-N1 component recorded over the lateral temporal cortex for tones and speech sounds.

Hayes et al. (16) examined the plasticity of the central auditory pathway in children with learning problems using a standard ABR paradigm and cortical potentials. They employed an auditory training program for a group of students with learning problems and compared them to a group of normal control students using brainstem and cortical-evoked potentials. The results demonstrated that the trained group improved and exhibited changes in cortical responses and exhibited no changes in the classical $\mathrm{ABR}$ in comparison to the control group.

Fujioka et al. (17) studied auditory long latency potential before and after musical training in children aged 4 to 6 years. They observed higher P1 amplitudes in children after musical training, which suggested an improvement in the neuronal circuit after this training regime.

Of the many types of auditory-evoked potentials used in assessing (C)APD, the MLR has attracted the most attention. Jerger and Johnson (18) have described the MLR as potentially the single most important auditory-evoked response in terms of its ability to help identify and understand (C)APD. For instance, MLR abnormalities have been found in children with learning, speech, and/or language disabilities $(18,19)$. The MLR is also affected in adults with (C)APD (20) and cortical lesions (21).

MLR appears to be a clinically usable sensitive indicator of central nervous system disorders involving the auditory system; however, there is still a paucity of data on the nature of MLR abnormalities in children with (C)APD and on how auditory training may affect this auditory potential. Therefore, the purpose of this study was to evaluate the possible effects of a comprehensive auditory training program on the amplitude, latency, and/or morphology of the MLR waves of children with (C)APD.

\section{Subjects and Methods}

This project was approved by the Institutional Review Board of Hospital das Clínicas, School of Medicine, São Paulo University (Protocol No. 712/02). The parents of the children participating in the study signed a written informed consent before the start of the evaluations.

\section{Subjects}

Thirty individuals with (C)APD [the (C)APD group] and 22 individuals without (C)APD [the control group] between 8 and 14 years of age, matched for gender and age, were used as subjects for this study. All subjects had a normal otoscopic examination and normal middle ear function consisting of a Type A tympanogram with a peak middle ear pressure between -99 and +50 daPa. All subjects also had normal hearing sensitivity, which was defined as pure tone thresholds between 0 - and 20-dB hearing level $(\mathrm{HL})$ at octave frequencies of 250, 500, 1000, 2000, 4000, and $8000 \mathrm{~Hz}$ and a normal overall IQ on the WISC test. None of the subjects had a medical history of neurologic pathology, head trauma, or drug use, or any language problems at the time of evaluation as determined by a speech-language pathologist.

The control group was free of learning disabilities (attention-deficit disorder, attention-deficit hyperactivity disorder, or other specific learning disabilities) based on school records and demonstrated normal academic performance. The control group achieved normal performance for both ears in all four tests of central auditory function (described subsequently).

The (C)APD group had no speech or language problems (verbal) as determined by a speech and language pathologist; however, the (C)APD group did suffer from learning difficulties as determined by the classroom teacher. In addition, subjects in the (C)APD group were categorized as such by their performance on the central auditory test battery. In order to meet the (C)APD classification, all subjects had to perform below normal in at least one ear for two of the four tests on the behavioral central auditory test battery $(22,23)$.

\section{Procedures}

All children in the study were tested in two separate sessions. All testing was conducted with the subject seated in a double-walled soundproofed booth. Pure tone hearing tests were conducted using the modified HughsonWestlake technique (24) with a Grason-Stadler GSI-61 audiometer. Middle ear function was assessed bilaterally with a Grason-Stadler GSI-33 middle ear analyzer using conventional clinical techniques.

A maximum of four central auditory tests were administered to each group in order to evaluate differences in (central) auditory processing ability and to access the different levels of the central auditory nervous system (CANS) (6). The four tests that were conducted (two monotic and two dichotic) were selected from the following test procedures: the pediatric speech intelligibility (PSI) test (25), speech-in-noise test (26), staggered spondaic word test (SSW) (27), dichotic digits test (28), and a dichotic nonverbal test (26). All central auditory tests were recorded onto a compact disc (CD). The CD was played on a Sony $C D$ player and routed through the audiometer 
to TDH-50 earphones. In some cases, certain tests were selected in place of others due to the age of the subjects and/or the ability of the subjects to respond reliably. All test items were presented at a $50-\mathrm{dB}$ sensation level (pure tone average).

Following the behavioral procedures, the subjects were prepared for MLR testing. Electrodes were placed on the right and left earlobes (inverting); on the forehead, Fpz (ground), and at the left and right parietal areas, C3 and C4 (non-inverting) (A1 = stimulus left ear, A2 = stimulus right ear). Impedance across the electrode array was less than $7 \mathrm{k} \Omega$ for all subjects. A commercial physiologic averager (Biologic Traveler Express) was used to generate the acoustic stimuli and to record neuro-electrical activity from the scalp from which the MLR waveform was derived.

A $70-\mathrm{dB} \mathrm{nHL}$ rarefaction polarity $100-\mu$ s click stimulus was presented through TDH-39 earphones at a rate of 9.8 clicks/s to each subject. The order of presentation to the subjects' right and left ears was randomized.

Neuro-electrical activity was picked-up by scalp electrodes and filtered using 20 - to $1500-\mathrm{Hz}$ analog band-pass filters with a 12-dB/oct roll-off. Waveforms were also post hoc filtered from 20 to $200 \mathrm{~Hz}$ to help clarify the waveform. The averaged waveform was composed of two replications of 1000 sweeps each, which were displayed on a 70-ms time window for reading and analysis. During MLR assessment, children were kept awake, seated in a comfortable chair, and watched silent cartoon videos presented on a TV monitor. We did not accept waves that showed more than a $10 \%$ rejection (29).

\section{Data analysis}

Peak-to-peak amplitudes and peak latencies were analyzed for all stimulus conditions. Amplitudes were measured from the most negative $\mathrm{Na}$ trough to the most positive peak of $\mathrm{Pa}$. The $\mathrm{Pa}$ peak was also used to determine the latency index. To be considered for Na response, latency had to occur between 14 and $25 \mathrm{~ms}$, and to be considered a $\mathrm{Pa}$, latency had to fall within the range of 21 to $45 \mathrm{~ms}$. Both descriptive and inferential statistics were used to analyze the data. Analysis of variance was used to determine the main effects of group (i.e., (C)APD group vs control group) and another analyses of variance were used to determine the auditory training effects (i.e., pre vs post for the (C)APD group). Measurements included Na$\mathrm{Pa}$ amplitude and $\mathrm{Pa}$ latency components. For behavioral central auditory test results, scores for left and right ears were averaged and statistical analyses were applied.

\section{Auditory training procedures}

The control subjects were tested with the behavioral and electrophysiological test procedures at the same time as the (C)APD group, but were not enrolled in the auditory training program. After the initial assessment, the (C)APD group was enrolled for two months of auditory training.
They were retested one month after the end of the training (three months after the first test). The auditory training was both formal and informal and consisted of sessions of 50 min each once a week over an 8-week period. The types of training were equally distributed in regard to time. Not all training procedures were completed in one session. In addition, the subjects with (C)APD received informal training with their parents at home for 15 min each day.

The formal training was summarized and consisted of the following procedures based on Musiek and Schochat (30).

Frequency training. This training technique involves the discrimination of two different tones (low and high). Step 1: the subjects had to determine if two tones were the same or different. Step 2: the subjects had to label the two tones they heard by pitch, either high-low or low-high. Step 3: the stimuli included three tones that varied in pitch and the subject had to relay the correct sequence, i.e., high $(\mathrm{H})$-low (L)-high $(\mathrm{H}), \mathrm{LLH}, \mathrm{HHL}$, etc.

The interstimulus interval and tone duration were varied in accordance with the subjects' performances. Overall, the therapist tried to keep subject performance in the $30-80 \%$ correct performance range. The stimuli were presented at a comfortable listening level.

Intensity training. Intensity training was similar to the frequency training technique, wherein the intensities of the tones were changed instead of the frequencies.

Temporal training. Temporal training was similar to frequency and intensity training but instead focused on the duration of the tone. We also used gap detection to train temporal processing. Gap detection training consisted of listening to segments of white noise that contained silent gaps. The subjects were required to press a response button whenever a gap was heard. The occurrence of the gap in the noise was randomized and the gap duration was varied systematically depending on the subjects' performance.

Dichotic interaural intensity difference. This auditory training technique has been described elsewhere $(21,30,31)$, and therefore, will be described only briefly here. This technique requires an asymmetry of dichotic performance. The intensity level in the better (or stronger) ear is decreased and the level in the poorer ear is maintained, usually around 50-dB HL, until the poorer ear's performance is close to normal. Because the stronger ear is receiving less intensity, its performance will usually decrease approximately as much as the poorer ear's performance increases. Therapy starts at the intensity level that allows the performance of the poor ear to be near normal. Using a variety of dichotic materials, the subject listens and reports the dichotic stimuli. High performance is maintained in the poor ear while (using an adaptive approach) the intensity in the strong ear is gradually increased (increasing the performance of that ear). Once both ears reach near normal dichotic performance, therapy 
is stopped; however, in this study, the subjects continued the training for the specified time period regardless of performance.

Localization and speech perception. The subjects have to listen to speech signals and competing signals inside the booth in four different positions: 1) speakers at each ear, 2) speakers in front and back of the head, 3) speakers at each ear in the opposite position of condition one, and 4) speakers in front and back of the head in the opposite position of condition two. Position changes were obtained by having the subject turn their chair 90 degrees for every listening condition. Target stimuli were words presented through one speaker and competing stimuli (white noise, speech babble) through the other. The target words were presented at $50-\mathrm{dB} \mathrm{HL}$ and competing stimuli were presented at varying levels depending on the performances of the subjects.

Informal training. Informal training was done at home with the parents $15 \mathrm{~min}$ a day. Parents were oriented to the informal training techniques, which included the following steps: 1) the subject listened to a story and raised his/ her hand every time he/she heard one target word; 2) the subject had to listen to a story and sketch each paragraph. After 4 or 5 paragraphs (sketches), the subject had to recount the story based on his/her drawings; 3) a topic was selected (e.g., fruit, clothes, and animals) and the subject was asked to add a word. For example, parents say apple and the subject has to say apple and add another fruit; 4) listen to songs and repeat the lyrics.

The subject was given directions starting with one-step directives, which were increased to 4-step directives. These directives involved motor tasks so the subject could be easily monitored (21).

Parents were required to keep records of the progress of their child in these tasks. Not all informal procedures were performed at each therapy session. The therapy techniques that were omitted in a given session were included in the following session.

\section{Results}

\section{Behavioral auditory processing tests}

Although it was not the purpose of this study, we would like to report results on the behavioral tests for the (C)APD group for pre- and post-training. There was a significant improvement in performance after therapy for the (C)APD group for each test (Table 1). A series of one-way ANOVAs found differences $(\mathrm{P}<0.001)$ between pre- and post-auditory training conditions for all four behavioral tests for the (C)APD group. The number of control subjects in retests varied from that at the initial testing since some children could not attend the follow-up test session. All subjects in the (C)APD group returned for follow-up MLR and for behavioral testing. The data in Table 1 depict the average of both ears. The $\mathrm{N}$ number in Tables 2, 3, 4, and 5 varied because some of the children did not show $\mathrm{Pa}$ waves at some of the electrode sites.

\section{Electrophysiology}

A representative MLR tracing from one patient of the (C)APD group is presented in Figure 1, wherein the $\mathrm{Na}$, $\mathrm{Pa}$, and $\mathrm{Pb}$ peaks are labeled. Tracing showed pre- and post-training recordings for the two electrode sites and both ears.

\section{MLR tracing pre- and post-training}

One-way ANOVA results for the MLR from the control group showed no statistically significant difference between first test and follow-up test for either amplitude or latency for all electrode sites (data not shown).

Aone-way ANOVA showed no statistically significant difference between pre- and post-auditory training conditions

Table 1. Effect of training on the behavioral test results of the (C)APD group.

\begin{tabular}{|c|c|c|c|c|c|c|c|c|}
\hline \multirow[t]{2}{*}{ Pre vs Post } & \multicolumn{2}{|c|}{ PSI (s/r - 15) } & \multicolumn{2}{|c|}{ Speech-in-noise } & \multicolumn{2}{|c|}{ Non-verbal dichotic } & \multicolumn{2}{|c|}{ SSW/Digits } \\
\hline & Pre & Post & Pre & Post & Pre & Post & Pre & Post \\
\hline Mean & $70.8 \%$ & $82.2 \%$ & $72.9 \%$ & $86.0 \%$ & $71.9 \%$ & $93.2 \%$ & $63.5 \%$ & $78.0 \%$ \\
\hline Median & $70.0 \%$ & $80.0 \%$ & $76.0 \%$ & $88.0 \%$ & $79.0 \%$ & $100.0 \%$ & $63.8 \%$ & $80.0 \%$ \\
\hline SD & $20.2 \%$ & $13.0 \%$ & $16.7 \%$ & $7.2 \%$ & $24.9 \%$ & $10.5 \%$ & $19.1 \%$ & $14.9 \%$ \\
\hline $\mathrm{N}$ & 48 & 50 & 48 & 50 & 48 & 50 & 48 & 50 \\
\hline$P$ & \multicolumn{2}{|c|}{$0.001^{*}$} & \multicolumn{2}{|c|}{$<0.001^{*}$} & \multicolumn{2}{|c|}{$<0.001^{*}$} & \multicolumn{2}{|c|}{$<0.001^{*}$} \\
\hline
\end{tabular}

Both ears were averaged together for these statistics. The $\mathrm{N}$ reflects both ears, and varies slightly across conditions because not all patients received the same tests. $P \leq 0.001$, Pre mean compared to Post mean for each test (descriptive statistics and ANOVA). (C)APD = (central) auditory processing disorder; Pre = before auditory training; Post $=$ after auditory training; $\mathrm{PSI}=$ pediatric speech intelligibility test; SSW = staggered spondaic word test; Digits = dichotic digits test; $\mathrm{SD}=$ standard deviation; $\mathrm{N}$ = sample size . 
in the latency for the (C)APD group; however, a significant difference in the amplitudes for the electrodes placed at $\mathrm{C} 3$ for both ears was noted. Tables 2 and 3 provide descrip- tive statistics and ANOVA values for latency and amplitude measures, respectively.

Comparisons were also made between (C)APD and

Table 2. Comparison of latency values (in $\mathrm{ms}$ ) for the (C)APD group before and after training.

\begin{tabular}{|c|c|c|c|c|c|c|c|c|}
\hline \multirow[t]{2}{*}{ (C)APD-latency } & \multicolumn{2}{|c|}{ C3-A1 } & \multicolumn{2}{|c|}{ C4-A1 } & \multicolumn{2}{|c|}{ C3-A2 } & \multicolumn{2}{|c|}{ C4-A2 } \\
\hline & Pre & Post & Pre & Post & Pre & Post & Pre & Post \\
\hline Mean & 34.24 & 34.97 & 35.94 & 36.11 & 35.70 & 35.50 & 34.26 & 33.77 \\
\hline Median & 33.93 & 35.29 & 36.46 & 36.27 & 35.93 & 35.88 & 34.32 & 33.54 \\
\hline SD & 4.27 & 2.96 & 4.04 & 2.93 & 3.74 & 3.22 & 4.47 & 3.04 \\
\hline $\mathrm{N}$ & 30 & 30 & 29 & 30 & 29 & 29 & 28 & 29 \\
\hline$P$ & \multicolumn{2}{|c|}{0.443} & \multicolumn{2}{|c|}{0.847} & \multicolumn{2}{|c|}{0.827} & \multicolumn{2}{|c|}{0.623} \\
\hline
\end{tabular}

Variations in $\mathrm{N}$ across conditions were the result of unmeasurable middle latency responses in some cases. None of the Pre/Post comparisons of means were statistically different (descriptive statistics and ANOVA). (C)APD = (central) auditory processing disorder; $P r e=$ before auditory training; Post = after auditory training; $S D=$ standard deviation; $\mathrm{N}=$ sample size .

Table 3. Comparison of amplitude values (in $\mu \mathrm{V}$ ) for the (C)APD group before and after training.

\begin{tabular}{|c|c|c|c|c|c|c|c|c|}
\hline \multirow[t]{2}{*}{ (C)APD-amplitude } & \multicolumn{2}{|c|}{ C3-A1 } & \multicolumn{2}{|c|}{ C4-A1 } & \multicolumn{2}{|c|}{ C3-A2 } & \multicolumn{2}{|c|}{ C4-A2 } \\
\hline & Pre & Post & Pre & Post & Pre & Post & Pre & Post \\
\hline Mean & 0.84 & 1.59 & 1.01 & 1.33 & 0.69 & 1.24 & 0.96 & 1.18 \\
\hline Median & 0.76 & 1.44 & 0.97 & 1.02 & 0.63 & 0.98 & 0.78 & 1.13 \\
\hline SD & 0.39 & 0.82 & 0.51 & 1.36 & 0.31 & 0.73 & 0.60 & 0.53 \\
\hline $\mathrm{N}$ & 30 & 30 & 29 & 30 & 29 & 29 & 28 & 29 \\
\hline$P$ & \multicolumn{2}{|c|}{$<0.001^{*}$} & \multicolumn{2}{|c|}{0.235} & \multicolumn{2}{|c|}{$<0.001^{*}$} & \multicolumn{2}{|c|}{0.149} \\
\hline
\end{tabular}

Variations in $\mathrm{N}$ across conditions were a result of unmeasurable middle latency responses in some cases. $\mathrm{P} \leq 0.001$, Pre mean compared to Post mean (descriptive statistics and ANOVA). $(C) A P D=($ central $)$ auditory processing disorder; Pre $=$ before auditory training; Post $=$ after auditory training; $\mathrm{SD}=$ standard deviation; $\mathrm{N}$ = sample size.

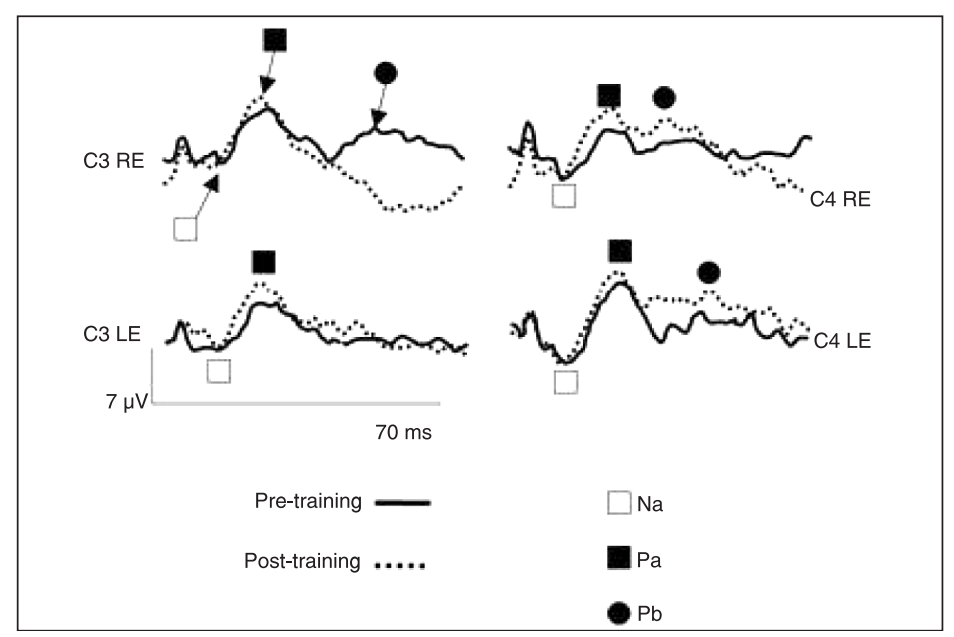

Figure 1. Comparison of middle latency response before and after auditory training of all subjects of the (C)APD group. RE = right ear; $L E=$ left ear. 
Table 4. Comparison of latency values (in ms) for (C)APD and control (normal) groups before and after training.

\begin{tabular}{|c|c|c|c|c|c|c|c|c|}
\hline \multirow[t]{2}{*}{ Latency } & \multicolumn{2}{|c|}{ C3-A1 } & \multicolumn{2}{|c|}{ C4-A1 } & \multicolumn{2}{|c|}{ C3-A2 } & \multicolumn{2}{|c|}{ C4-A2 } \\
\hline & Normal & (C)APD & Normal & (C)APD & Normal & (C)APD & Normal & (C)APD \\
\hline \multicolumn{9}{|l|}{ Before } \\
\hline Mean & 35.49 & 34.24 & 35.19 & 35.94 & 35.85 & 35.70 & 34.87 & 34.26 \\
\hline Median & 35.10 & 33.93 & 35.49 & 36.46 & 33.93 & 35.93 & 34.03 & 34.32 \\
\hline SD & 2.46 & 4.27 & 2.67 & 4.04 & 4.73 & 3.74 & 3.55 & 4.47 \\
\hline $\mathrm{N}$ & 22 & 30 & 22 & 29 & 22 & 29 & 22 & 28 \\
\hline $\mathrm{P}$ & \multicolumn{2}{|c|}{0.221} & \multicolumn{2}{|c|}{0.453} & \multicolumn{2}{|c|}{0.895} & \multicolumn{2}{|c|}{0.603} \\
\hline \multicolumn{9}{|l|}{ After } \\
\hline Mean & 35.17 & 34.97 & 35.22 & 36.11 & 35.67 & 35.50 & 34.89 & 33.77 \\
\hline Median & 34.90 & 35.29 & 34.42 & 36.27 & 35.00 & 35.88 & 33.93 & 33.54 \\
\hline SD & 4.91 & 2.96 & 4.51 & 2.93 & 4.88 & 3.22 & 5.12 & 3.04 \\
\hline $\mathrm{N}$ & 22 & 30 & 22 & 30 & 22 & 29 & 22 & 29 \\
\hline$P$ & \multicolumn{2}{|c|}{0.860} & \multicolumn{2}{|c|}{0.392} & \multicolumn{2}{|c|}{0.878} & \multicolumn{2}{|c|}{0.334} \\
\hline
\end{tabular}

Variations in $\mathrm{N}$ across conditions were a result of unmeasurable middle latency responses in some cases. None of the comparisons of normal vs (C)APD means were statistically different before or after training (descriptive statistics and ANOVA). (C)APD = (central) auditory processing disorder; $\mathrm{SD}=$ standard deviation; $\mathrm{N}=$ sample size .

Table 5. Comparison of amplitude values (in $\mu \mathrm{V}$ ) for (C)APD and control (normal) groups before and after training.

\begin{tabular}{|c|c|c|c|c|c|c|c|c|}
\hline \multirow[t]{2}{*}{ Amplitude } & \multicolumn{2}{|c|}{ C3-A1 } & \multicolumn{2}{|c|}{ C4-A1 } & \multicolumn{2}{|c|}{ C3-A2 } & \multicolumn{2}{|c|}{ C4-A2 } \\
\hline & Normal & (C)APD & Normal & (C)APD & Normal & (C)APD & Normal & (C)APD \\
\hline \multicolumn{9}{|l|}{ Before } \\
\hline Mean & 1.18 & 0.84 & 1.48 & 1.01 & 1.00 & 0.69 & 1.18 & 0.96 \\
\hline Median & 0.92 & 0.76 & 1.14 & 0.97 & 0.91 & 0.63 & 1.00 & 0.78 \\
\hline SD & 0.65 & 0.39 & 0.77 & 0.51 & 0.46 & 0.31 & 0.66 & 0.60 \\
\hline $\mathrm{N}$ & 22 & 30 & 22 & 29 & 22 & 29 & 22 & 28 \\
\hline$P$ & \multicolumn{2}{|c|}{$0.022^{*}$} & \multicolumn{2}{|c|}{$0.010^{*}$} & \multicolumn{2}{|c|}{$0.005^{*}$} & \multicolumn{2}{|c|}{0.230} \\
\hline \multicolumn{9}{|l|}{ After } \\
\hline Mean & 1.26 & 1.59 & 1.09 & 1.33 & 1.20 & 1.24 & 1.07 & 1.18 \\
\hline Median & 1.29 & 1.44 & 0.94 & 1.02 & 1.04 & 0.98 & 0.84 & 1.13 \\
\hline SD & 0.59 & 0.82 & 0.66 & 1.36 & 0.63 & 0.73 & 0.55 & 0.53 \\
\hline $\mathrm{N}$ & 22 & 30 & 22 & 30 & 22 & 29 & 22 & 29 \\
\hline $\mathrm{P}$ & \multicolumn{2}{|c|}{0.115} & \multicolumn{2}{|c|}{0.457} & \multicolumn{2}{|c|}{0.825} & \multicolumn{2}{|c|}{0.496} \\
\hline
\end{tabular}

Variations in $\mathrm{N}$ across conditions were a result of unmeasurable middle latency responses in some cases. ${ }^{*} P<0.03$, Normal compared to (C)APD means before training (descriptive statistics and ANOVA). None of the comparisons of Normal vs (C)APD means were statistically different after training (descriptive statistics and ANOVA). (C)APD = (central) auditory processing disorder; SD = standard deviation; $\mathrm{N}=$ sample size. 
control groups. For latency measures made before and after training, no differences were found between control and (C)APD groups. These results are shown in Table 4.

For amplitude measures, differences were noted between control and (C)APD groups before training for all electrode sites except C4 for the right ear (Table 5); however, differences were not found between the two groups after training for any electrode site, as can be seen in Table 5 .

\section{Discussion}

The results of this study have demonstrated an improvement in the MLR-evoked response after the auditory training applied in this group of children diagnosed with (C)APD. Although behavioral tests of central auditory function can be used to measure the influences of auditory training, auditory-evoked potentials provide a relatively objective and sensitive index of neurophysiologic alterations related to training (32). It is because of this, and the fact that there is a paucity of data on MLR and (C)APD, that this evoked potential was employed in this study. It is also known that the likely generators of the MLR are neurons in the thalamocortical areas of the brain, which is a neural substrate likely involved in central auditory processing (33).

It seems clear that lesions of the CANS will compromise the MLR $(21,34)$. It is not as clear that the MLR may be abnormal in children with learning disabilities (LD) or suspected of (C)APD. Purdy et al. (4) and Arehole et al. (35) have reported slight alterations in the amplitude and latency of the MLR, respectively, in these pediatric populations. Specifically, Purdy et al. (4) showed the Na wave to be slightly longer in a group of children with LD than in controls. In addition, the $\mathrm{Nb}$ amplitude was less negative for the LD group. Arehole et al. (35) showed delays in the $\mathrm{Pa}$ wave for the LD population but no effect on amplitude.

Although Klein et al. (15) found more normal ABRs and MLRs in their group of patients with verbal auditory agnosia than we found in ours, we can state that our patients have a different kind of auditory alteration, meaning that most likely their patients have more cortical deficits and most likely ours have deficits in the neurons of the thalamo-cortical areas.

In the present study, one key finding was that there was a significant difference in the $\mathrm{Na}-\mathrm{Pa}$ amplitudes between (C)APD and control groups in three of the four test conditions. The most affected electrode site was C3 (left hemisphere). To our knowledge, this finding has not been reported before under these circumstances. Similar MLR results have been reported in individuals with CANS disorders, but not in studies on (C)APD. Similar to the present study, it has been well documented that in confirmed lesions of CANS, MLR amplitudes are affected more than latencies $(21,34)$. Therefore, the lack of a latency difference in the conducted analysis was not surprising.

It is of interest that training appeared to have its greatest influence on the $\mathrm{C} 3$ recording site. It is difficult to determine if the effect was related to factors within the population or to the types of training that were performed. It is known that the MLR has a long maturational course (12). Perhaps the children with (C)APD require a longer period of time for their MLR generators to mature. In this regard, there is evidence of reduced callosal input to the left hemisphere in children with (C)APD for dichotic listening tasks (36). This reduced left hemisphere input could be associated with the maturation of myelin in the corpus callosum and in related pathways. A lack of callosal input to the left hemisphere has been shown in secondary topographic mapping to possibly be the result of degeneration (demyelination) of the corpus callosum in relation to aging (37). In addition, one of the training procedures employed in this study was dichotic interaural intensity difference training. This procedure is designed to enhance the function of callosal and corticocallosal connections in the brain $(21,32)$. Hence, although speculative at this point, these are some of the possible mechanisms that could underlie the left hemisphere findings observed in this study.

The fact that MLR amplitudes did change after training is similar to findings reported elsewhere in studies of auditory training and evoked potentials. Improved speech discrimination subsequent to training has been reflected in MMN results in the work by Kraus et al. (12). Additionally, Tremblay and associates $(9,38)$ have shown that auditory training in speech syllables improves the perception of these sounds and enhances the N1-P2 auditory-evoked response to these speech sounds. The auditory training employed by Kraus et al. (12) and Tremblay and colleagues $(9,38)$ was more specific than that used in the present study. The training in the present study was broad and used a variety of auditory-training procedures. Nonetheless, significant electrophysiological changes were noted. We conclude that further studies are needed.

The finding that there were no significant differences between the pre-and post-conditions for either amplitude or latency in the control group is important because it indicates stability of the MLR to Pa waves for test-retest performance. This is important because these data suggest that the MLR may be a clinically valuable tool to identify (C)APD. It is also interesting that there was essentially no difference between groups in regard to MLR amplitude after training. Hence, it could be argued that auditory training improved the MLR primarily for the indices that were initially responsible for the difference between groups.

Although it was not the objective of this study, behavioral central auditory test results for the (C)APD group revealed improvements in scores of all tests applied after training. Again, this is similar to other studies, which have also shown improvements in auditory behaviors that were correlated to electrophysiological enhancement, presumably from auditory training $(2,9,12,38)$. Improvements in auditory behavior after training were consistent across all behavioral tests. This result may reflect the nonspecific, 
broad auditory training methods that were used in the current study. These findings could also indicate that a wide variety of auditory training approaches and lengths may serve to improve auditory performance. In this regard, it is interesting to note that, in the literature, a variety of auditory training methods have been employed that have resulted in improvements in auditory-evoked potentials. For example, Jirsa (2) used a general auditory training program for a 7-week period (14 sessions), and demonstrated an increased P300 amplitude in children suspected of (C)APD. Additionally, Tremblay et al. (9) used specific synthetic speech for training and testing over the course of 10 days, wherein their subjects demonstrated an increase in amplitude of the N1-P2 complex. It is difficult to determine if specific or nonspecific auditory training paradigms will yield the best results for either behavioral or electrophysiological measures; however, what does seem to be emerging is that auditory training can influence, in a positive way, physiologic and behavioral responses related to audition. There is even some evidence that auditory enhancement from auditory training may generalize to listening situations well beyond those of the training paradigm (10).

Musiek and Berge (39) pointed out that changes in the auditory system with appropriate auditory training have

\section{References}

1. Kraus N, McGee TJ, Carrell TD, Zecker SG, Nicol TG, Koch DB. Auditory neurophysiologic responses and discrimination deficits in children with learning problems. Science 1996; 273: 971-973.

2. Jirsa RE. The clinical utility of the P3 AERP in children with auditory processing disorders. J Speech Hear Res 1992; 35: 903-912.

3. Musiek FE, Gollegly KM, Kibbe KS, Verkest SB. Current concepts on the use of $A B R$ and auditory psychophysical tests in the evaluation of brain stem lesions. Am J Otol 1988; 9 (Suppl): 25-35.

4. Purdy SC, Kelly AS, Davies MG. Auditory brainstem response, middle latency response, and late cortical evoked potentials in children with learning disabilities. J Am Acad Audiol 2002; 13: 367-382.

5. American Speech-Language-Hearing Association. (Central) Auditory Processing Disorders [Technical Report]; 2005. www.asha.org/docs/html/TR2005-00043.html. Accessed June 29, 2010.

6. Jerger J, Musiek F. Report of the Consensus Conference on the Diagnosis of Auditory Processing Disorders in SchoolAged Children. J Am Acad Audiol 2000; 11: 467-474.

7. Martin BA, Tremblay KL, Korczak P. Speech evoked potentials: from the laboratory to the clinic. Ear Hear 2008; 29: 285-313.

8. Tremblay KL. Training-related changes in the brain: evidence from human auditory-evoked potentials. Semin Hear 2007; 28: 120-132.

9. Tremblay K, Kraus N, McGee T, Ponton C, Otis B. Central auditory plasticity: changes in the N1-P2 complex after been postulated for many years, but experimental evidence in humans has been slow to confirm these changes. In contrast, for animal models, these plastic changes have been shown for many years.

The MLR is an evoked potential procedure that has been recommended as a test measure for (C)APD (6). It is a clinically feasible procedure and is generated by auditory areas of the brain that may be involved in (C)APD. In this study, the MLR to the Na-Pa wave amplitude was smaller in children with (C)APD than in the control group. The auditory-training regimen applied in this study increased the $\mathrm{Na}-\mathrm{Pa}$ amplitude of the MLR and increased performance in behavioral tests of auditory processing.

These findings suggest progress in the use of electrophysiological measurements in the diagnosis and treatment of (C)APD. These results also contribute to the emerging body of literature that supports the use of auditory training for those with (C)APD. Future studies are planned to determine if these electrophysiological and behavioral changes are maintained over long periods of time.

\section{Acknowledgments}

\author{
Research supported by FAPESP (\#02/06651-9).
}

speech-sound training. Ear Hear 2001; 22: 79-90.

10. Tremblay K, Kraus N, Carrell TD, McGee T. Central auditory system plasticity: generalization to novel stimuli following listening training. J Acoust Soc Am 1997; 102: 3762-3773.

11. Kraus N. Speech sound perception, neurophysiology, and plasticity. Int J Pediatr Otorhinolaryngol 1999; 47: 123-129.

12. Kraus N, McGee T, Carrell T, Sharma A, Nicol T. Mismatch negativity to speech stimuli in school-age children. Electroencephalogr Clin Neurophysiol Suppl 1995; 44: 211-217.

13. Tremblay K, Kraus N, McGee T. The time course of auditory perceptual learning: neurophysiological changes during speech-sound training. Neuroreport 1998; 9: 3557-3560.

14. Menning $\mathrm{H}$, Roberts LE, Pantev $\mathrm{C}$. Plastic changes in the auditory cortex induced by intensive frequency discrimination training. Neuroreport 2000; 11: 817-822.

15. Klein SK, Kurtzberg D, Brattson A, Kreuzer JA, Stapells DR, Dunn MA, et al. Electrophysiologic manifestations of impaired temporal lobe auditory processing in verbal auditory agnosia. Brain Lang 1995; 51: 383-405.

16. Hayes EA, Warrier CM, Nicol TG, Zecker SG, Kraus N. Neural plasticity following auditory training in children with learning problems. Clin Neurophysiol 2003; 114: 673-684.

17. Fujioka T, Ross B, Kakigi R, Pantev C, Trainor LJ. One year of musical training affects development of auditory cortical-evoked fields in young children. Brain 2006; 129: 2593-2608

18. Jerger J, Johnson K. Interactions of age, gender, and sensorineural hearing loss on ABR latency. Ear Hear 1988; 9: 168-176.

19. Milicic D, Alcada MN, Pais CL, Vecerina-Volic S, Jurkovic J, 
Pais CM. A study of auditory afferent organization in children with dyslalia. Int J Pediatr Otorhinolaryngol 1998; 46: 4356.

20. Marvel JB, Jerger JF, Lew HL. Asymmetries in topographic brain maps of auditory-evoked potentials in the elderly. J Am Acad Audiol 1992; 3: 361-368.

21. Musiek FE, Baran JA, Schochat E. Selected management approaches to central auditory processing disorders. Scand Audiol Suppl 1999; 51: 63-76.

22. Chermak GD, Musiek FE. Central auditory processing disorders. San Diego: Singular Publishing Group San Diego; 1997.

23. American Speech-Language-Hearing Association. (Central) Auditory Processing Disorders; 2005. www.asha.org/policy. Accessed June 29, 2010.

24. Carhart R, Jerger J. Preferred method for clinical determination of pure tone thresholds. J Speech Hear Disord 1959; 24: 330-345.

25. Jerger S, Jerger S. The Pediatric Speech Intelligibility Test (PSI). St. Louis: Auditec of St. Louis; 1984.

26. Pereira LD, Schochat E. Processamento auditivo central: manual de avaliação. São Paulo: Lovise; 1997.

27. Katz J. The SSW test: an interim report. J Speech Hear Disord 1968; 33: 132-146.

28. Musiek FE. Assessment of central auditory dysfunction: the dichotic digit test revisited. Ear Hear 1983; 4: 79-83.

29. Thornton RD. Instrumental and recording parameters in auditory-evoked potentials. In: Burkard RF, Don M, Eggermont JJ (Editors), Auditory evoked potentials: Basic principles and clinical application. Baltimore: Lipincott Willians \& Wilkins; 2007.
30. Musiek FE, Schochat E. Auditory training and central auditory processing disorders. Sem Hear 1998; 19: 357-366.

31. Musiek FE, Shinn J, Hare C. Plasticity, auditory training and auditory processing disorders. Sem Hear 2002; 23: 263275.

32. Merzenich MM, Jenkins WM, Johnston P, Schreiner C, Miller SL, Tallal P. Temporal processing deficits of languagelearning impaired children ameliorated by training. Science 1996; 271: 77-81.

33. Kraus N, McGee T, Carrell T, King C, Littman T, Nicol T. Discrimination of speech-like contrasts in the auditory thalamus and cortex. J Acoust Soc Am 1994; 96: 2758-2768.

34. Kileny P, Paccioretti D, Wilson AF. Effects of cortical lesions on middle-latency auditory evoked responses (MLR). Electroencephalogr Clin Neurophysiol 1987; 66: 108-120.

35. Arehole S, Augustine LE, Simhadri R. Middle latency response in children with learning disabilities: preliminary findings. J Commun Disord 1995; 28: 21-38.

36. Musiek FE, Kibbe K, Baran JA. Neuroaudiological results from split-brain patients. Sem Hear 1984; 5: 219-230.

37. Jerger J, Alford B, Lew H, Rivera V, Chmiel R. Dichotic listening, event-related potentials, and interhemispheric transfer in the elderly. Ear Hear 1995; 16: 482-498.

38. Tremblay KL, Kraus N. Auditory training induces asymmetrical changes in cortical neural activity. J Speech Lang Hear Res 2002; 45: 564-572.

39. Musiek FE, Berge BE. A neuroscience view of auditory training/stimulation and central auditory processing disorders. In: Masters MG, Stecker NA, Katz J (Editors), Central auditory - processing disorders mostly management. Boston: Allyn and Bacon; 1988. p 15-24. 